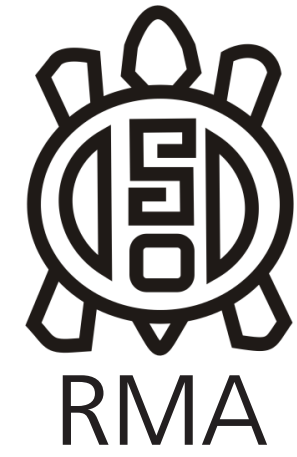

Dossier

\title{
Análisis de núcleos de obsidiana del Holoceno tardío en el centro-oeste de la provincia de Santa Cruz
}

\author{
Analysis of obsidian cores of the late Holocene from central-west \\ Santa Cruz province
}

Gisela Cassiodoro*, Agustín Agnolin** y Josefina Flores Coni***

\begin{abstract}
*Consejo Nacional de Investigaciones Científicas y Técnicas, Instituto Nacional de Antropología y Pensamiento Latinoamericano, Universidad de Buenos Aires, Argentina. E-mail: gcassio@hotmail.com

** Consejo Nacional de Investigaciones Científicas y Técnicas, Instituto Nacional de Antropología y Pensamiento Latinoamericano, Argentina. E-mail: agusagnolin@yahoo.com.ar *** Consejo Nacional de Investigaciones Científicas y Técnicas, Instituto Nacional de Antropología y Pensamiento Latinoamericano, Argentina. E-mail: josefinafloresconi@gmail.com
\end{abstract}

\begin{abstract}
Resumen
Los núcleos constituyen uno de los artefactos que permiten estudiar las características del abastecimiento de rocas, el proceso inicial de reducción y la producción de formas base. De este modo, se evalúan las características de la utilización de la obsidiana negra de Pampa del Asador, localizada en el centro-oeste de la provincia de Santa Cruz, durante el Holoceno tardío. Para tal fin se realiza un análisis comparativo de las características de los núcleos de obsidiana negra recuperados en la fuente de aprovisionamiento de Pampa del Asador y en sectores localizados a diferentes distancias de la misma, como la cuenca del lago Salitroso, las mesetas del Guitarra y el Strobel y la cuenca del lago Cardiel. Se considera el grado y tipo de reducción de los núcleos tomando en cuenta variables como frecuencia, estado, dimensiones, tipos de núcleos y presencia de corteza. Se concluye que la distancia a la fuente no explica todos los patrones observados, contribuyendo a la discusión sobre las estrategias involucradas en el transporte y explotación de la obsidiana en una escala espacial amplia.
\end{abstract}

Palabras clave: Obsidiana; Núcleos; Explotación; Distancia; Patagonia.

\begin{abstract}
Cores allow studying the characteristics of raw material supply, the initial process of reduction and the production of blanks. In this way, we evaluate the use of black obsidian cores from Pampa del Asador in the central-western province of Santa Cruz during late Holocene. To this end, we develop a comparative analysis of the properties of obsidian cores recovered from the supply source of Pampa del Asador and from sectors located at different distances from it, such as Salitroso and Cardiel lake basins and Guitarra and Strobel plateau. We considered the degree and type of reduction of the cores taking into account variables such as frequencies, dimensions, types of cores and presence of cortex. Results show that distance does not explain the observed patterns. Thus, the strategies involved in the transport and exploitation of obsidian are discussed.
\end{abstract}

Keywords: Obsidian, Cores, Explotation, Distance, Patagonia.

\section{Introducción}

Para el Holoceno tardío las investigaciones referidas a tecnología lítica en el centro-oeste de la provincia de Santa Cruz han abordado la utilización de las materias primas y el uso del espacio en el marco de condiciones ambientales de desecación regional (Bozzuto, 2013; Cassiodoro, 2011; Espinosa, 2002; Flores Coni, 2018; entre otros). Dichas investigaciones se han centrado en dar cuenta de las estrategias tecnológicas desarrolladas en cada uno de los diferentes sectores ambientales que caracterizan a la región: cuencas lacustres bajas y mesetas basálticas y cuencas lacustres altas. Considerando que dentro de este espacio se localiza la fuente regional de obsidiana negra en Pampa del Asador (Espinosa y Goñi, 1999), en esta oportunidad buscamos ahondar en las características del transporte y utilización de este recurso lítico a partir del estudio de la distribución y análisis de atributos morfológicos de núcleos en una escala espacial amplia. De esta forma, se realiza un análisis comparativo de piezas recuperadas, no solo en inmediaciones a Pampa del Asador (PDA), sino también en sectores localizados a diferentes distancias de la misma, como la cuenca del lago Salitroso, las mesetas del Guitarra y Strobel y la cuenca 


\section{del lago Cardiel (Figura 1).}

Desde una perspectiva de la organización tecnológica (sensu Nelson, 1991), los núcleos constituyen uno de los artefactos que permiten estudiar las características del abastecimiento de rocas, el proceso inicial de reducción y la producción de formas base (Andrefsky, 1998; Paulides, 2006, entre otros). Asimismo, para comprender las trayectorias de uso de materias primas es pertinente incluir información sobre las particularidades de las fuentes de abastecimiento (Ericson, 1984), ya que la distancia a la misma y el modo en que se presenta el recurso pueden influir en las estrategias tecnológicas desarrolladas (Bamforth, 1986). De este modo, entendemos que ambas variables pueden condicionar las características y distribución de los núcleos (Andrefsky, 1998; Paulides, 2006).

La PDA es una planicie glacifluvial de $1200 \mathrm{~km}^{2}$ de extensión que se caracteriza por tener depósitos de acarreo con una alta disponibilidad de sílice, basalto y, principalmente, obsidiana negra (Espinosa y Goñi,

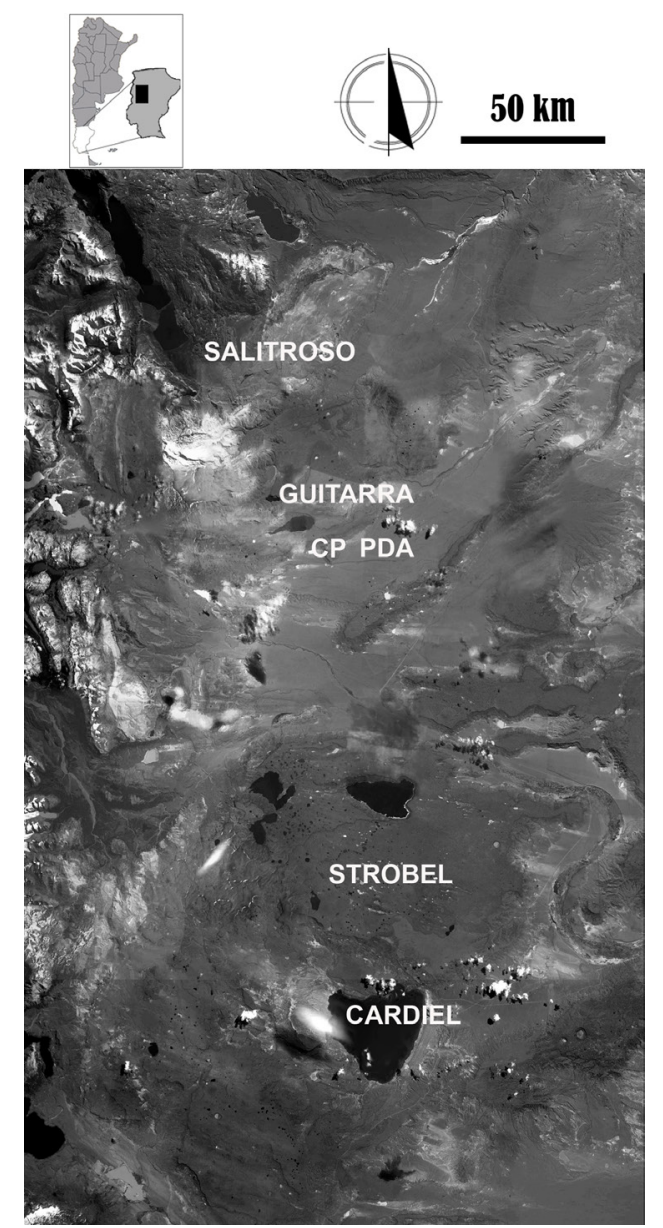

Figura 1: Área de estudio. Referencias: CP: Cerro Pampa; PDA: Pampa del Asador.

Figure 1: Study area. References: CP: Cerro Pampa; PDA: Pampa del Asador.
1999). Esta última materia prima se presenta en forma de guijarros de tamaños variables que van entre los 24 y los $122 \mathrm{~mm}$ de largo máximo, lo que los hace fácilmente transportables. En términos espaciales, la obsidiana se distribuye a lo largo de toda la PDA pero existen sectores donde los guijarros se encuentra en mayor frecuencia, tal como las inmediaciones del Cerro Pampa (Cassiodoro, Goñi, Espinosa, Agnolin y Flores Coni 2017).

Las particularidades de esta materia prima y su extendido uso en Patagonia meridional la hacen un caso de relevancia para evaluar las estrategias de transporte y explotación empleadas por los grupos de cazadores-recolectores en el Holoceno tardío. Así, en este trabajo se parte de la expectativa que la distancia sería un factor importante en las estrategias de explotación de los recursos líticos. De este modo, se espera que a mayor distancia a PDA se den una serie de características distintivas en los núcleos y conjuntos, entre las que se destacan: una disminución en la frecuencia y dimensiones de los núcleos, una mayor frecuencia de núcleos agotados y fracturados, una menor presencia de corteza y estrategias de reducción que maximicen el número de lascas/hojas extraídas por pieza (por ejemplo, presencia de núcleos bipolares y con extracciones paralelas).

\section{Materiales y métodos}

En el marco de discutir las estrategias involucradas en el transporte y explotación de la obsidiana en la región de estudio, la variable que guía inicialmente el problema de investigación en este trabajo es la distancia a la fuente de abastecimiento. Por lo tanto, la muestra abordada está constituida por núcleos recuperados a diferentes distancias y orientaciones de PDA. Las distancias son consideradas en términos lineales desde uno de los puntos en los que se registra la mayor densidad de guijarros de obsidiana, el Cerro Pampa (Figura 1). Los materiales provienen de sitios localizados en las inmediaciones de este cerro, de sectores localizados al norte del mismo, como la meseta del lago Guitarra y la cuenca del lago Salitroso, y otros localizados hacia el sur como la meseta del lago Strobel y la cuenca del lago Cardiel. En todos los casos fueron seleccionados aquellos conjuntos asignables al Holoceno tardío.

En función de la distancia, se considera la frecuencia, el grado y tipo de reducción de los núcleos evaluando variables como el estado, las dimensiones, la presencia de corteza, piezas agotadas y tipos de núcleos. En primer lugar, no solo se contó la cantidad de núcleos de obsidiana, sino también su relación con la frecuencia total de núcleos en cada sitio. Respecto a las dimensiones, se consideró el volumen de piezas enteras multiplicando largo, ancho y espesor, expresado en $\mathrm{cm}^{3}$. Para evaluar el grado de reducción, también se tomó en cuenta información publicada sobre las dimensiones de guijarros naturales de obsidiana de PDA (Cassiodoro et al., 2017) 
y de los depósitos de la localidad 17 de Marzo (Franco, Brook, Cirigliano, Stern y Vetrisano, 2017). En esta última, localizada a unos $170 \mathrm{~km}$ al sudeste de PDA, a unos $130 \mathrm{~km}$ del Strobel y 120 del Cardiel, se han registrado depósitos con escasos guijarros de obsidiana, de dimensiones menores, pero con la misma señal geoquímica de PDA (Franco et al., 2017). Asimismo, se consignó la presencia de corteza en los núcleos enteros y la cantidad de piezas agotadas. Estas últimas son aquellas que no permiten nuevas extracciones, en base a su tamaño y la presencia de charnelas. Por su parte, para aportar información inicial sobre el carácter de las extracciones efectuadas, se consideró el tipo de núcleo a partir de la propuesta de Aschero $(1975,1983)$ y usando categorías generales de Andrefsky (1998) y Stiner y Kuhn (1992). Así, se toma en consideración que núcleos centrípetos o con múltiples extracciones multidireccionales pueden buscan maximizar el tamaño de lascas, mientras que aquellos con extracciones paralelas (incluidos los núcleos de hojas) estarían vinculados con la posibilidad de maximizar el número de extracciones por pieza (Stiner y Kuhn, 1992). Por su parte, núcleos bipolares han sido relacionados con la maximización de la materia prima y las dimensiones de los guijarros naturales (Flegenheimer, Bayón y González De Bonaveri, 1995).

\section{Características de los núcleos de obsidiana}

Respecto a la frecuencia, el primer punto a destacar es que en todos los sitios que tienen núcleos de obsidiana, hay más de una unidad (Tabla 1). A su vez, como es esperable, en los sectores más próximos a la fuente de aprovisionamiento la representación de núcleos de obsidiana ( $r$-0.75; valor $p: 0,13)$ y la cantidad de núcleos por sitio ( $r$ : $-0,72$; valor $p: 0,16)$ es mayor (Tabla 1$)$. No obstante, en ambos casos la correlación es moderada y no es estadísticamente significativa. En términos del eje de dirección, se observa que existe una mayor frecuencia de núcleos en los espacios localizados al norte de PDA, tales como la meseta del lago Guitarra y la cuenca del lago Salitroso en contraposición a los espacios hacia el sur (Tabla 1). Solo se han recuperado guijarros naturales de obsidiana en los sitios de Cerro Pampa y del Cardiel.
En todos los sectores predominan los núcleos enteros $y$, como es esperable, Cerro Pampa tiene los mayores porcentajes $(81,62 \%)$, seguido por Guitarra $(78,26 \%)$ y Cardiel (76,2\%). En la cuenca del lago Salitroso (37,31\%) y en la meseta del Strobel $(33,4 \%)$ es donde hay una mayor representación de piezas fracturadas (ver Tabla 1 para total de núcleos y Tabla 2 para total de piezas enteras).

Respecto a las dimensiones, Cerro Pampa tiene los tamaños más grandes de núcleos enteros (Tabla 2, Figura 2 A). El tamaño máximo de los núcleos de Cerro Pampa es casi la mitad del tamaño máximo de los guijarros naturales de PDA y el $75 \%$ de las piezas con tamaños mayores supera los $44,4 \mathrm{~cm}^{3}$. Si se comparan los volúmenes de los guijarros de PDA y los núcleos de los sitios más próximos a ella, es decir Cerro Pampa y Guitarra, se observa que hay diferencias estadísticamente significativas (test KruskalWallis $\mathrm{H}: 63,02, \mathrm{p}:<0,05)$. Es decir que en la fuente los guijarros son muy procesados, lo que lleva a que se modifique sustancialmente el volumen del núcleo. En los sitios de Cerro Pampa, junto con los núcleos también se registraron 11 guijarros naturales cuyo volumen promedio es de $128,4 \mathrm{~cm}^{3}$. Por su parte, en Strobel el $25 \%$ de las piezas menores tiene $4,52 \mathrm{~cm}^{3}$ o menos, esto hace que sea el sector con tamaños más reducidos. No obstante, como también tiene tamaños más grandes, es el sector con mayor variabilidad de tamaños (CV: 198,27). Salitroso también tiene piezas pequeñas $\left(25 \%\right.$ menor a $\left.6,79 \mathrm{~cm}^{3}\right)$ pero una menor variación de tamaños ya que los núcleos no superan los $34,2 \mathrm{~cm}^{3}$. Por su parte, Cardiel es el que sigue a Cerro Pampa en términos del tamaño promedio de núcleos y, de manera similar a Salitroso, el 25\% de las piezas menores no superan un volumen de $6,5 \mathrm{~cm}^{3}$. En esta cuenca se registró la presencia de 4 guijarros naturales, en menos de $1 \mathrm{~m}^{2}$, con volúmenes que van entre los 76,4 y $258,3 \mathrm{~cm}^{3}$. Finalmente, el volumen promedio de los guijarros de 17 de marzo es mucho menor a cualquiera de los valores de los sectores en estudio (Tabla 2).

De las piezas enteras, tanto en Cerro Pampa ( $\mathrm{n}$ : 111) como en Cardiel y Strobel ( $\mathrm{n}$ : 16 cada una), los porcentajes de núcleos con corteza superan el $80 \%$. En Salitroso (n:

\begin{tabular}{|c|c|c|c|c|c|c|c|}
\hline Área & $\begin{array}{l}\text { Distancia } \\
\text { promedio } \\
\text { en km a } \\
\text { PDA }\end{array}$ & $\begin{array}{c}\text { Sitios } \\
\text { muestreados }\end{array}$ & $\begin{array}{l}\text { Sitios con } \\
\text { núcleos de } \\
\text { obsidiana }\end{array}$ & $\begin{array}{l}\text { Núcleos } \\
\text { obsidiana }\end{array}$ & $\begin{array}{c}\text { Total } \\
\text { núcleos }\end{array}$ & $\begin{array}{c}\text { \% núcleos } \\
\text { de } \\
\text { obsidiana }\end{array}$ & $\begin{array}{c}\text { Núcleos de } \\
\text { obsidiana por } \\
\text { sitio }\end{array}$ \\
\hline Cerro Pampa & 0 & 3 & 3 & 136 & 180 & 75,56 & 45,33 \\
\hline Guitarra & 10 & 6 & 5 & 46 & 73 & 63,01 & 9,2 \\
\hline Salitroso & 50 & 24 & 11 & 67 & 177 & 37,85 & 6,1 \\
\hline Strobel & 70 & 83 & 15 & 24 & 75 & 32,00 & 1,6 \\
\hline Cardiel & 100 & 31 & 9 & 21 & 164 & 12,80 & 2,33 \\
\hline \multicolumn{2}{|c|}{ Total } & 147 & 43 & 294 & 669 & 43,9 & 6,8 \\
\hline
\end{tabular}

Tabla 1: Distancia a Pampa del Asador y frecuencia de núcleos de obsidiana.

Table 1: Distance to Pampa del Asador and frequency of obsidian cores. 
42) estos valores alcanzan el $74 \%$. Por su parte, dada la cercanía a PDA, llama la atención que en Guitarra (n: 36) el porcentaje de núcleos enteros con corteza apenas superen el $50 \%$ de la muestra. Si se consideran las dimensiones de estos núcleos con corteza (Figura 2 B), resalta la variabilidad de tamaños de Cerro Pampa, esperable en un contexto inmediatamente próximo a los depósitos naturales. En Cardiel y Strobel dicha variabilidad queda sobredimensionada por la representación de un núcleo que supera los $214 \mathrm{~cm}^{3}$, en cada caso. Más allá de la variabilidad, el volumen típico de los núcleos enteros con corteza (Figura 2 B) es mayor y similar en Cerro Pampa (mediana: $27 \mathrm{~cm}^{3}$ ) y Guitarra (mediana: $24,2 \mathrm{~cm}^{3}$ ). Por su parte, Salitroso (mediana: $10,1 \mathrm{~cm}^{3}$ ) y Strobel (mediana: 10,3 $\mathrm{cm}^{3}$ ) tienen valores menores y similares. La muestra más distante a PDA, el Cardiel (mediana: 15,1 $\mathrm{cm}^{3}$ ), presenta valores mayores a los de estos dos últimos sectores.

Los núcleos de obsidiana recuperados en la región tienden a tener aún posibilidades de uso. No obstante, se destaca un mayor porcentaje de núcleos agotados sobre el total de núcleos en la meseta del Strobel (33,3\%; N: 24). Asimismo, los porcentajes de núcleos agotados son similares en sectores a distintas distancias de PDA como la cuenca del Cardiel (19\%; N: 21), el Salitroso (19,4\%; N: 67) y Cerro Pampa (18,4\%; N: 136). La meseta del Guitarra tiene los valores menores (10,8\%; N: 46).

En la muestra analizada existe un claro predominio de la técnica de extracción de lascas ya que solo se ha registrado un núcleo de hojas en la meseta del Guitarra. Además de la presencia de núcleos con extracciones paralelas (Tabla 3), en este espacio también existe una alta representación de piezas con extracciones centrípetas. Esta variabilidad de modalidad de reducción de núcleos también se reconoce en Cerro Pampa, registrándose tanto núcleos bipolares como aquellos con lascados aislados. Estos últimos podrían estar relacionados con la realización de pruebas en la materia prima y las primeras etapas de descortezamiento de guijarros. Por su parte, en Strobel se destacan los porcentajes de núcleos con extracciones paralelas y bipolares, dado que ambos tipos podrían estar vinculados con estrategias que busquen maximizar el recurso lítico. En Salitroso también se destacan los núcleos bipolares pero la representación de núcleos centrípetos supera el 50\% de la muestra (Tabla 3). Finalmente, en la cuenca del lago Cardiel, la modalidad de reducción de núcleo es menos variable ya que más del $80 \%$ son núcleos centrípetos.

\section{Discusión}

En la provincia de Santa Cruz, diferentes investigaciones buscan evaluar aspectos de la movilidad y circulación de los grupos cazadores-recolectores en función de la frecuencia de obsidiana negra de PDA en los conjuntos (Civalero, 1999; Cueto, Frank y Skarbun, 2017; Pallo y Borrero, 2015; entre otros). Particularmente, en este análisis sobre las características morfológicas de los núcleos buscamos ahondar en las estrategias de explotación de la obsidiana. Tomando en conjunto las variables abordadas se evidencia el desarrollo de diferentes patrones de transporte y reducción de núcleos en los distintos sectores considerados.

En primer lugar, los conjuntos artefactuales de Cerro Pampa están en inmediaciones a los depósitos en los que afloran los guijarros de obsidiana negra, por lo cual la existencia de guijarros en los sitios y las características de los núcleos son concordantes con lo que se esperaría en una fuente de aprovisionamiento, tareas de descortezamiento inicial, producción de formas base,

Tabla 2: Volumen de núcleos y guijarros naturales enteros en $\mathrm{cm}^{3}$. Referencias: N: total núcleos enteros, D.E.: Desviación estándar, Q1: primer cuartil, Q3: tercer cuartil, C.V: Coeficiente de variación.

Table 2: Volume of complete cores and natural pebbles in $\mathrm{cm}^{3}$. References: N: total complete cores, D.E.: Standard deviation, Q1: first quartile, Q3: third quartile, C.V: Coefficient of variation.

\begin{tabular}{|l|c|c|c|c|c|c|c|}
\hline \multirow{2}{*}{} & \multicolumn{2}{|c|}{ Guijarros naturales } & \multicolumn{4}{c|}{ Núcleos } \\
\cline { 2 - 8 } & PDA & 17 de Marzo & $\begin{array}{c}\text { Cerro } \\
\text { Pampa }\end{array}$ & Guitarra & Salitroso & Strobel & Cardiel \\
\hline $\mathrm{N}$ & 57 & 25 & 111 & 36 & 42 & 16 & 16 \\
\hline Mínimo & 6,48 & 0,90 & 1,96 & 2,00 & 2,21 & 2,96 & 3,00 \\
\hline Máximo & 845,25 & 70,18 & 472,97 & 46,44 & 34,20 & 214,14 & 250,00 \\
\hline Media & 116,47 & 9,90 & 41,15 & 19,50 & 11,76 & 26,67 & 32,00 \\
\hline D.E. & 146,77 & 13,77 & 53,58 & 10,40 & 7,76 & 52,88 & 59,70 \\
\hline Q1 & 45,43 & 3,38 & 17,00 & 10,56 & 6,79 & 4,52 & 6,50 \\
\hline Q3 & 118,35 & 11,03 & 44,40 & 25,54 & 14,77 & 16,37 & 29,50 \\
\hline Mediana & 65,29 & 6,26 & 24,99 & 17,78 & 9,70 & 8,75 & 16,50 \\
\hline CV & 126,01 & 139,01 & 130,19 & 53,33 & 65,98 & 198,27 & 186,55 \\
\hline
\end{tabular}




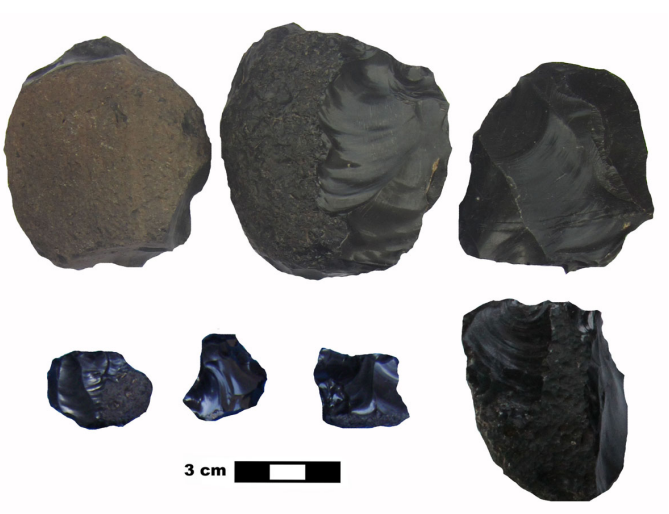

A

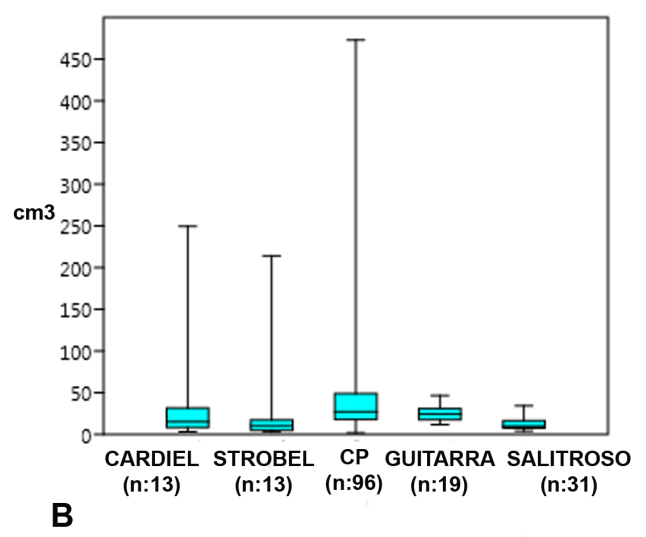

Figura 2: A: Núcleos del área de estudio. B: Dimensiones de núcleos enteros con corteza. Referencias: CP: Cerro Pampa.

Figure 2: A: Cores from the study area. B: Dimensions of complete cores with cortex. References: CP: Cerro Pampa.

Tabla 3: Tipos de núcleos. Referencias: c/ls: con lascados.

Table 3: Types of cores. References: c/ls: with knappings.

\begin{tabular}{|c|c|c|c|c|c|c|}
\hline Tipo & Cerro Pampa & Guitarra & Salitroso & Strobel & Cardiel & Total \\
\hline c/ls aislados & 26,13 & - & 2,38 & 6,25 & - & 31 \\
\hline centrípetos & 31,53 & 61,11 & 59,52 & 31,25 & 81,25 & 100 \\
\hline paralelos & 36,94 & 25 & 19,05 & 43,75 & 12,50 & 67 \\
\hline bipolares & 5,41 & 8,33 & 16,67 & 12,50 & - & 18 \\
\hline indeterminados & - & 5,56 & 2,38 & 6,25 & 6,25 & 5 \\
\hline Total & $100(\mathrm{n}: 111)$ & $100(\mathrm{n}: 36)$ & $100(\mathrm{n}: 42)$ & $100(\mathrm{n}: 16)$ & $100(\mathrm{n}: 16)$ & 221 \\
\hline
\end{tabular}

existencia de pruebas, etc. (Andrefsky, 1998; Ericson, 1984; Paulides, 2006). Asimismo, se registra variabilidad en la reducción de los mismos ya que no solo existen piezas escasamente utilizadas, sino también núcleos agotados y piezas bipolares. A escasos $10 \mathrm{~km}$ al norte se encuentran los conjuntos de la meseta del Guitarra que dan cuenta del transporte de núcleos parcialmente descortezados y con una exigua explotación. En la cuenca del Salitroso, a unos $50 \mathrm{~km}$ en la misma dirección, también se transportan núcleos parcialmente descortezados pero el aprovechamiento de los mismos ha sido intenso. Un patrón similar ocurre en la meseta del Strobel localizada a unos $70 \mathrm{~km}$ de PDA, pero hacia el sur. A diferencia de lo que ocurre en Salitroso, en este caso los núcleos pudieron haber sido transportados totalmente descortezados. En oposición a lo que ocurre en Salitroso y Strobel, en el sector más distante aquí abordado, la cuenca del lago Cardiel, aunque el flujo del recurso es menor, se han transportado guijarros enteros y núcleos parcialmente descortezados. Las dimensiones de los guijarros sin utilizar son solo vinculables con las de los guijarros de PDA. Asimismo, el hecho de que se localicen en un punto muy específico de la cuenca, lleva a pensar en que forman parte de un escondrijo de materia prima. Por su parte, el aprovechamiento de los núcleos en esta cuenca es parcial.

De este modo, puede observarse variabilidad en las estrategias involucradas en el transporte y explotación de la obsidiana en una escala espacial amplia. En principio, la distancia a la fuente de aprovisionamiento no explica inequívocamente todos los patrones observados. Aunque puede estar interviniendo en el flujo de obsidiana a los diferentes espacios, no necesariamente tiene influencia en el aprovechamiento que se da a este recurso lítico. En parte, el hecho que la obsidiana se presente en forma de guijarros de dimensiones que no llegan a los $900 \mathrm{~cm}^{3}$ puede estar favoreciendo su transporte tal cual se encuentra en la naturaleza a sectores distantes como la cuenca del lago Cardiel. Sin embargo, más allá de la portabilidad del recurso, los patrones de descarte y explotación siguen siendo variables en la región. Por lo tanto, múltiples factores pueden estar interactuando tales como la utilización diferencial de los sectores considerados (espacios residenciales versus logísticos), la disponibilidad de otros recursos líticos (por ejemplo, limolita en Cardiel), las estrategias de aprovisionamiento desarrolladas (directas o indirectas), etc. Asimismo, junto con la distancia, algunos de estos factores también pueden estar influyendo en la mayor representación de núcleos de obsidiana en los espacios localizados al norte de PDA considerados en este trabajo. De este modo, resulta necesario seguir con la problemática planteada abordando nuevas variables morfológicas, así como incorporar aspectos no considerados en este trabajo como los porcentajes de corteza en los núcleos. Por otra parte, resulta necesario a futuro integrar los resultados obtenidos con los provenientes de los artefactos formatizados y desechos de talla.

En síntesis, a partir de este acercamiento a los núcleos de obsidiana del centro-oeste de Santa Cruz consideramos relevante no sobredimensionar la importancia de la distancia a la fuente de aprovisionamiento para dar cuenta del aprovechamiento dado a este recurso lítico. Asimismo, 
se ha podido establecer el desarrollo de diferentes estrategias de explotación de núcleos de obsidiana en los distintos espacios considerados durante el Holoceno tardío que merecen ser profundizadas en futuros trabajos.

Buenos Aires, 27 de marzo de 2019

\section{Agradecimientos}

Las investigaciones fueron subsidiadas por los proyectos PICT 2013-1965, UBACyT 2018-2020 20020170100150BA, PIP-CONICET N¹1220120100406 y N¹1220120100442. Se agradece la colaboración de la municipalidad de Gobernador Gregores y Perito Moreno, Goldcorp y los propietarios y empleados de las diferentes estancias. Asimismo, a los comentarios de los evaluadores y los participantes y coordinadores del simposio.

\section{Bibliografía}

Aschero, C. (1975). Ensayo para una clasificación morfológica de artefactos líticos aplicada a estudios tipológicos comparativos. Informe presentado al CONICET. Ms.

Aschero, C. (1983). Ensayo para una clasificación morfológica de artefactos líticos. Apéndice $A$ y $B$. Cátedra de Ergología y Tecnología. Facultad de Filosofía y Letras, UBA. Ms.

Andrefsky, W. (1998). Lithics Macroscopic approaches to analysis. Cambridge, UK: Cambridge University Press.

Bamforth, D. (1986). Technological efficiency and tool curation. American Antiquity, 51, 38-50.

Bozzuto, D. (2013). Análisis de material lítico superficial en el noreste del lago Pueyrredón-Cochrane (provincia de Santa Cruz, Argentina). Revista Española de Antropología Americana 43 (1), 73-88.

Cassiodoro, G. (2011). Movilidad y uso del espacio de cazadores-recolectores del Holoceno tardío: estudio de la variabilidad del registro tecnológico en distintos ambientes del noroeste de la provincia de Santa Cruz. Oxford, UK: British Archaeological Reports (International Series), Archaeopress.

Cassiodoro G., Goñi R., Espinosa S., Agnolin A. y Flores Coni J. (2017). Black obsidian from Pampa del Asador (Santa Cruz, Patagonia Argentina): a regional source. En Alberti, J., K. Borrazzo, S. Buscaglia, A. Castro Esnal, A. Elias, P. Brousse, D. Hereñú y P. L. Franco (Comps.) 11th International Symposium on Knappable Materials. From Toolstone to Stone Tools. Book of Abstracts (p. 91). Buenos Aires, Argentina: IMHICHU-CONICET.

Civalero, T. (1999). Obsidiana en Santa Cruz, una problemática a resolver. En J. Belardi, P. Fernández, R. Goñi,
G. Guráieb y M. De Nigris (Eds.), Soplando en el Viento. Actas de las III Jornadas de Arqueología de la Patagonia (pp. 155-164). Comahue, Neuquen: INAPL-UN del Comahue.

Cueto M., Frank, A. y Skarbun, F. (2017). The exploitation of obsidian in the Central Plateau of Santa Cruz, Argentina: Results from La María and Cerro Tres Tetas and a regional perspective. Journal of Lithic Studies 3 (2). doi:10.2218/jls.v3i2.1402.

Ericson, J. (1984). Toward the analysis of lithic reduction systems. En J. Ericson y B. Purdy (Eds.), Prehistoric Quarries and Lithic Producion (pp. 11-22). Cambridge, UK: Cambridge University Press.

Espinosa, S. (2002). Estrategias tecnológicas líticas y uso del espacio en momentos tardíos en el Parque Nacional Perito Moreno (Santa Cruz). (Tesis doctoral). Universidad de Buenos Aires, Buenos Aires, Argentina.

Espinosa, S. y Goñi, R. (1999). Viven! Una fuente de obsidiana en la Pcia. de Santa Cruz. En J. Belardi, P. Fernández, R. Goñi, G. Guráieb y M. De Nigris (Eds.), Soplando en el Viento. Actas de las III Jornadas de Arqueología de la Patagonia (pp. 177-188). Comahue, Neuquen: INAPL-UN del Comahue.

Flegenheimer, N., Bayón, C, y González De Bonaveri, M. (1995). Técnica simple, comportamientos complejos: la talla bipolar en la arqueología bonaerense. Relaciones de la Sociedad Argentina de Antropología XX, 81-110.

Flores Coni, J. (2018). Poblamiento humano y uso del espacio en la meseta del Strobel (provincia de Santa Cruz). Un análisis sobre la variabilidad tecnológica durante el Holoceno. (Tesis Doctoral). Universidad de Buenos Aires, Buenos Aires, Argentina.

Franco, N., Brook, G., Cirigliano, N., Stern, C. y Vetrisano, L. (2017). 17 de Marzo (Santa Cruz, Argentina): a new distal source of Pampa del Asador type black obsidian and its implications for understanding hunter-gatherer behavior in Patagonia. Journal Archaeological Science Report, 12, 232-243.

Nelson, M. (1991). The study of technological organization. Archaeological Method and Theory, 3, 57-100.

Pallo, C y Borrero, L. (2015). ¿Intercambio o movilidad?: una evaluación sobre el uso de escalas de análisis espaciales y curvas de declinación en Patagonia centro-meridional (Argentina). Latin American Antiquity, 26(3), 287-303.

Paulides, L. (2006). El núcleo de la cuestión. El análisis de los núcleos en los conjuntos líticos. En C. Pérez de Micou (Eds.), El modo de hacer las cosas. Artefactos y ecofactos en Arqueología (pp.: 67-101). Buenos Aires, Argentina: Universidad de Buenos Aires.

Stiner, M. y Kuhn, S. (1992). Subsistence, technology, and adaptive variation in Middle Paleolithic Italy. American Anthropologist, 94(2), 306-339. 